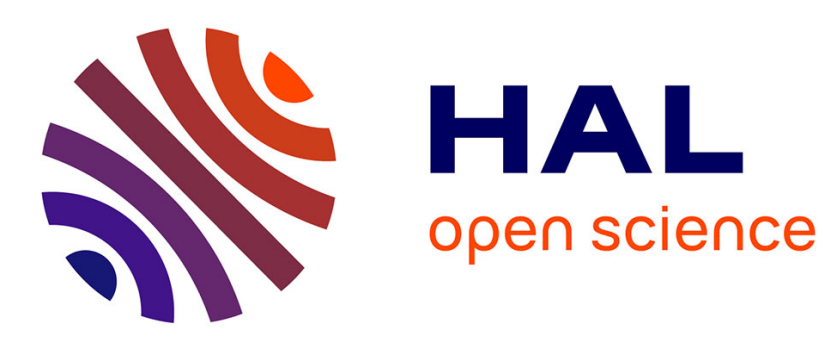

\title{
Mesure automatique de la diffusivité thermique
}

A. Degiovanni, M. Laurent, R. Prost

\section{To cite this version:}

A. Degiovanni, M. Laurent, R. Prost. Mesure automatique de la diffusivité thermique. Revue de Physique Appliquée, 1979, 14 (11), pp.927-932. 10.1051/rphysap:019790014011092700 . jpa00244681

\section{HAL Id: jpa-00244681 https://hal.science/jpa-00244681}

Submitted on 1 Jan 1979

HAL is a multi-disciplinary open access archive for the deposit and dissemination of scientific research documents, whether they are published or not. The documents may come from teaching and research institutions in France or abroad, or from public or private research centers.
L'archive ouverte pluridisciplinaire HAL, est destinée au dépôt et à la diffusion de documents scientifiques de niveau recherche, publiés ou non, émanant des établissements d'enseignement et de recherche français ou étrangers, des laboratoires publics ou privés. 


\title{
Mesure automatique de la diffusivité thermique
}

\author{
A. Degiovanni $\left({ }^{*}\right)$, M. Laurent $\left({ }^{*}\right)$ et R. Prost $\left({ }^{* *}\right)$ \\ (*) Laboratoire de Physique Industrielle \\ (**) Laboratoire d'Optique Corpusculaire et Ultrasons. \\ Institut National des Sciences Appliquées, 20, avenue A.-Einstein, 69621 Villeurbanne Cedex
}

(Reçu le 4 juillet, accepté le 18 septembre 1979)

\begin{abstract}
Résumé. - Le dispositif automatique de mesure de la diffusivité thermique qui est présenté utilise la méthode flash. Le modèle thermocinétique tient compte des pertes sur toutes les faces de l'échantillon. La diffusivité est obtenue à partir du thermogramme donnant l'évolution de la température sur la face de l'échantillon opposée à celle soumise au flash. Les variations de température sont détectées avec un thermo-couple à semi-conducteurs, sa f.é.m. est amplifiée par un dispositif qui réalise une compensation automatique de la tension de décalage due à la différence de température entre le bloc isotherme et l'échantillon. Un micro-ordinateur, construit à l'aide d'un microprocesseur, assure la mise en mémoire du thermogramme et le calcul de la diffusivité tout en garantissant une sécurité sur la qualité des résultats par l'émission éventuelle de messages d'erreurs et de grandeurs de contrôle. Ce dispositif donne une reproductibilité des mesures de l'ordre de $1 \%$.
\end{abstract}

\begin{abstract}
An automatic measuring system of the thermal diffusivity by the flash method is described. The thermocinetic model includes heat losses on all sides of the specimen. The thermal diffusivity is obtained from the temperature variations on the back of the side irradiated by the flash. These thermal changes are detected with a semi-conductor thermoelectric sensor. The e.m.f. is magnified by an amplifier system with automatic compensation of the offset voltage due to the temperature difference between the isothermal substrate and the specimen. A microcomputeur based on a microprocessor stores the signal and computes the diffusivity. It controls the measuring accuracy, gives intermediate data and sometimes error messages. If the measure is repeated, the results obtained only differ by $1 \%$.
\end{abstract}

Dans la conclusion de la Monographie du Bureau National de Métrologie : Thermocinétique Impulsionnelle et Mesure de la Diffusivité Thermique [1] étaient signalées les études effectuées en vue de réaliser des dispositifs comportant une chaîne d'acquisition numérique et un traitement par microprocesseur, pour afficher directement la valeur de la diffusivité.

Nous présentons dans ce texte un dispositif ainsi automatisé. Un peu moins d'un an d'exploitation a montré non seulement une amélioration quant à la rapidité et la simplicité d'utilisation, ce qui est un avantage primordial en mesures industrielles, mais également une meilleure reproductibilité des résultats, ce qui devrait intéresser les laboratoires de métrologie.

1. Modèle thermocinétique. - L'échantillon est cylindrique (Fig. 1); à sa surface le transfert de chaleur avec le milieu extérieur est caractérisé par trois coefficients d'échanges thermiques équivalents, indé-

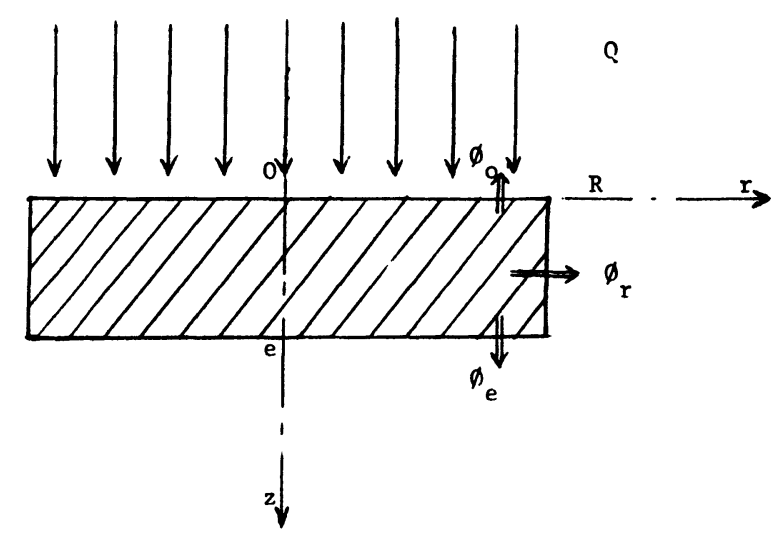

Fig. 1. - Schéma de principe.

[Cylindrical specimen.]

pendants de la température pendant la durée de l'expérience : $h_{0}$ sur la face $z=0, h_{\mathrm{e}}$ sur la face $z=e$ et $h_{\mathrm{R}}$ sur la face $r=R$. 
Le régime transitoire est alors solution du système suivant $[1,2]$ :

$$
\mid \begin{array}{ll}
\frac{1}{a} \frac{\partial T}{\partial t}=\frac{\partial^{2} T}{\partial r^{2}}+\frac{1}{r} \frac{\partial T}{\partial r}+\frac{\partial^{2} T}{\partial z^{2}} \\
\frac{\partial T}{\partial z}=\frac{h_{0}}{\lambda} T & \text { en } z=0 \\
\frac{\partial T}{\partial z}=-\frac{h_{\mathrm{e}}}{\lambda} T & \text { en } z=\mathrm{e} \\
\frac{\partial T}{\partial r}=-\frac{h_{\mathrm{R}}}{\lambda} T & \text { en } r=R \\
T=T_{0}(r, z) & \text { en } t=0 .
\end{array}
$$

Lorsque la condition initiale peut être écrite sous la forme :

$$
T=T_{0}(r, z)=f(r) . g(z)
$$

le système précédent se résout par séparation des variables, soit :

$$
T(r, z, t)=\sum_{n} \sum_{p} A_{n p} \cdot F_{p}(r) \cdot G_{n}(z) \cdot \exp \left(-V_{n p} \frac{a t}{e^{2}}\right) .
$$

Avec

$$
\begin{aligned}
A_{n p} & =\frac{A_{1} \cdot A_{2}}{A_{3} \cdot A_{4}} \\
A_{1} & =\int_{0}^{e} g(z) \cdot G_{n}(z) \mathrm{d} z \\
A_{2} & =\int_{0}^{R} r f(r) \cdot F_{p}(r) \mathrm{d} r \\
A_{3} & =\int_{0}^{e} G_{n}^{2}(z) \mathrm{d} z \\
A_{4} & =\int_{0}^{R} r F_{p}^{2}(r) \mathrm{d} r \\
F_{p}(r) & =J_{0}\left(\omega_{p} \frac{r}{R}\right) \\
G_{n}(z) & =\cos \left(u_{n} \frac{z}{e}\right)+\frac{h_{0} e}{\lambda u_{n}} \sin \left(u_{n} \frac{z}{e}\right) \\
V_{n p} & =u_{n}^{2}+\frac{e^{2}}{R^{2}} \omega_{p}^{2}
\end{aligned}
$$

où $u_{n}$ et $\omega_{p}$ sont solutions des équations transcendantes

$$
\mid \begin{aligned}
& \left(u^{2}-\frac{h_{0} h_{\mathrm{e}} e^{2}}{\lambda^{2}}\right) \operatorname{tg} u=\frac{\left(h_{0}+h_{\mathrm{e}}\right) e}{\lambda} u \\
& \omega . J_{1}(\omega)=\frac{h_{\mathrm{R}}}{\lambda} R . J_{0}(\omega)
\end{aligned}
$$

où $J_{0}$ et $J_{1}$ sont les fonctions de Bessel de première espèce d'ordre 0 et 1 .
2. Calcul de la diffusivité. - La face $z=0$ est soumise à une impulsion de flux infiniment courte uniformément répartie (nous discuterons plus loin des possibilités de prendre en considération la durée de l'impulsion); nous transposons cette condition de flux en condition de température en prenant pour origine des temps la fin de l'impulsion; l'éq. (6) devient :

$$
\begin{array}{lll}
g(z)=\frac{Q}{\varepsilon \rho c} & & 0 \leqslant z<\varepsilon \\
g(z)=0 & & \varepsilon \leqslant z \leqslant e \\
f(r)=1 & 0 \leqslant r \leqslant R
\end{array}
$$

avec

$Q$ énergie absorbée par unité de surface,

$\varepsilon \quad$ épaisseur infiniment petite absorbant l'énergie $Q$.

La solution en un point $r=r_{\mathrm{c}}$ de la face opposée à l'impulsion est donnée par l'éq. (7) qui devient :

$$
\begin{aligned}
& T^{*}\left(e, r_{\mathrm{c}}, t^{*}\right)= \\
& \quad=2 \sum_{n} \sum_{p} A_{n p} \cdot F_{p}\left(r_{\mathrm{c}}\right) \cdot G_{n}(e) \cdot \exp \left(-V_{n p} t^{*}\right)
\end{aligned}
$$

avec

$$
\begin{array}{r}
A_{n p}=\frac{u_{n}^{2}\left(u_{n}^{2}+L_{\mathrm{e}}^{2}\right)}{\left(u_{n}^{2}+L_{0}^{2}\right) \cdot\left(u_{n}^{2}+L_{\mathrm{e}}^{2}\right)+\left(L_{0}+L_{\mathrm{e}}\right)\left(u_{n}^{2}+L_{0} L_{\mathrm{e}}\right)} \times \\
\times \frac{2 L_{\mathrm{R}}}{\left(L_{\mathrm{R}}^{2}+\omega_{p}^{2}\right) \cdot J_{0}\left(\omega_{p}\right)}
\end{array}
$$

où

$$
\begin{aligned}
T^{*}= & T p c e / Q \text { (température réduite) } \\
t^{*}= & a t / e^{2} \quad \text { (temps réduit), } \\
L_{0}= & h_{0} e / \lambda, L_{\mathrm{R}}=h_{\mathrm{R}} R / \lambda, L_{\mathrm{e}}=h_{\mathrm{e}} e / \lambda \text { (coefficients } \\
& \text { d'échanges réduits). }
\end{aligned}
$$

Cette relation dépend alors de cinq paramètres :

$$
e / R, r_{\mathrm{c}} / R, L_{0}, L_{\mathrm{e}} \text { et } L_{\mathrm{R}}
$$

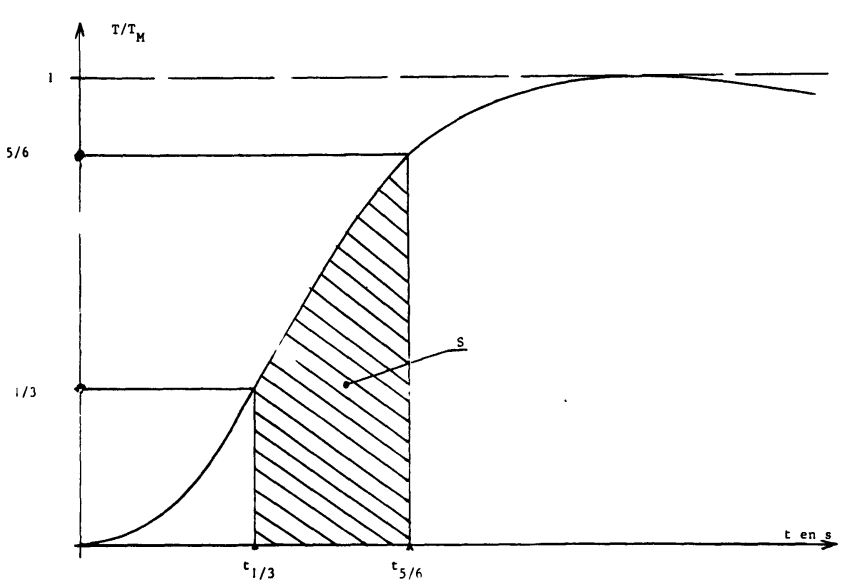

Fig. 2. - Courbe expérimentale.

[Experimental recording.] 


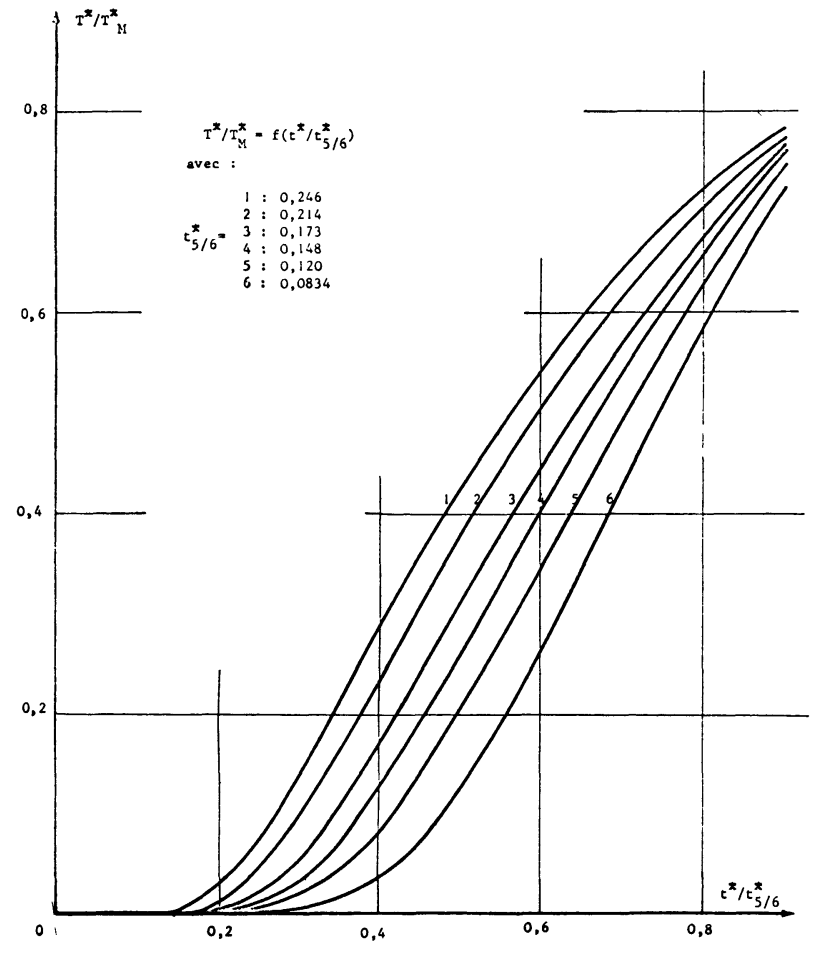

Fig. 3. - Courbes théoriques normalisées.

[Theoretical curves of temperature $T^{*} / T_{\mathrm{M}}^{*}$ versus $t^{*} / t_{5 / 6}^{*}$.]

en caractérisant ces courbes $T^{*}=f\left(t^{*}\right)$ par un temps réduit particulier $t_{\alpha}^{*}$ (abscisse du point dont l'ordonnée est $\alpha . T_{\mathrm{M}}^{*}$, avec $T_{\mathrm{M}}^{*}$ température maximale réduite), la diffusivité est donnée par :

$$
a=\frac{e^{2}}{t_{\alpha}} t_{\alpha}^{*}
$$

Pour déterminer le paramètre $t_{\alpha}^{*}$ sans faire appel à des simplifications, soit sur la répartition et la grandeur des échanges, soit sur la solution analytique, nous comparons l'enregistrement expérimental (Fig. 2) aux courbes théoriques (Fig. 3) après les avoir normalisées (nous utilisons respectivement les ordonnées du maximum $\left(T_{\mathrm{M}}\right.$ et $T_{\mathrm{M}}^{*}$ ) et les abscisses ( $t_{\alpha}$ et $\left.t_{\alpha}^{*}\right)$ des points dont les ordonnées sont $\left(\alpha T_{M}\right.$ et $\left.\alpha T_{M}^{*}\right)$ ).

Cette comparaison est possible car le réseau de courbes théoriques ainsi normalisées

$$
\left(T^{*} / T_{\mathrm{M}}^{*}=f\left(t^{*} / t_{\alpha}^{*}\right)\right)
$$

et paramétrées par $t_{\alpha}^{*}$ s'avère être indépendant des cinq autres paramètres $\left(e / R, r_{\mathrm{c}} / R, L_{0}, L_{\mathrm{e}}, L_{\mathrm{R}}\right)$.

Cette propriété n'est pas démontrée analytiquement, mais vérifiée numériquement sur un très large éventail de valeurs couvrant tous les cas réels et même au-delà :

avec

$$
0,1<e / R<10 \text { et } 0,01<r_{\mathrm{c}} / R<1
$$

$$
\begin{aligned}
& L_{0}=L_{\mathrm{e}}=L_{\mathrm{R}}=L \text { et } 0<L<20 \\
& L_{\mathrm{R}}=0 L_{\mathrm{e}}=L_{0}=L \text { et } 0<L<1, \\
& L_{\mathrm{R}}=L_{\mathrm{e}}=0 \text { et } 0<L_{0}<1
\end{aligned}
$$

La comparaison s'effectue par l'intermédiaire d'une intégrale définie des courbes normalisées, soit

$$
S^{*}=f\left(t^{*} / t_{\alpha}^{*}\right)
$$

avec :

$$
S^{*}=\frac{1}{T_{\mathrm{M}}^{*} \cdot t_{\alpha}^{*}} \int_{t_{\beta}^{*}}^{t_{\alpha}^{*}} T^{*} \mathrm{~d} t^{*}=\frac{1}{T_{\mathrm{M}} \cdot t_{\alpha}} \int_{t_{\beta}}^{t_{\alpha}} T \mathrm{~d} t=S / t_{\alpha} .
$$

En pratique, nous avons choisi $\alpha=5 / 6$ et $\beta=1 / 3$.

Le paramètre $t_{5 / 6}^{*}$ est alors donné (dans les limites $\left.0,09<t_{5 / 6}^{*}<0,252\right)$ par :

$$
t_{5 / 6}^{*}=-0,053+0,339 \frac{S}{t_{5 / 6}}+1,356\left(S / t_{5 / 6}\right)^{2}
$$

et la diffusivité par :

$$
a=\frac{e^{2}}{t_{5 / 6}} t_{5 / 6}^{*} .
$$

3. Appareillage (Fig. 4). - Nous ne décrirons pas en détail le dispositif de mesure (Fig. 4) et le générateur d'impulsion, ceci a été fait de façon détaillée dans la thèse de A. Degiovanni [2], ainsi que dans plusieurs articles $[3,4,5]$.

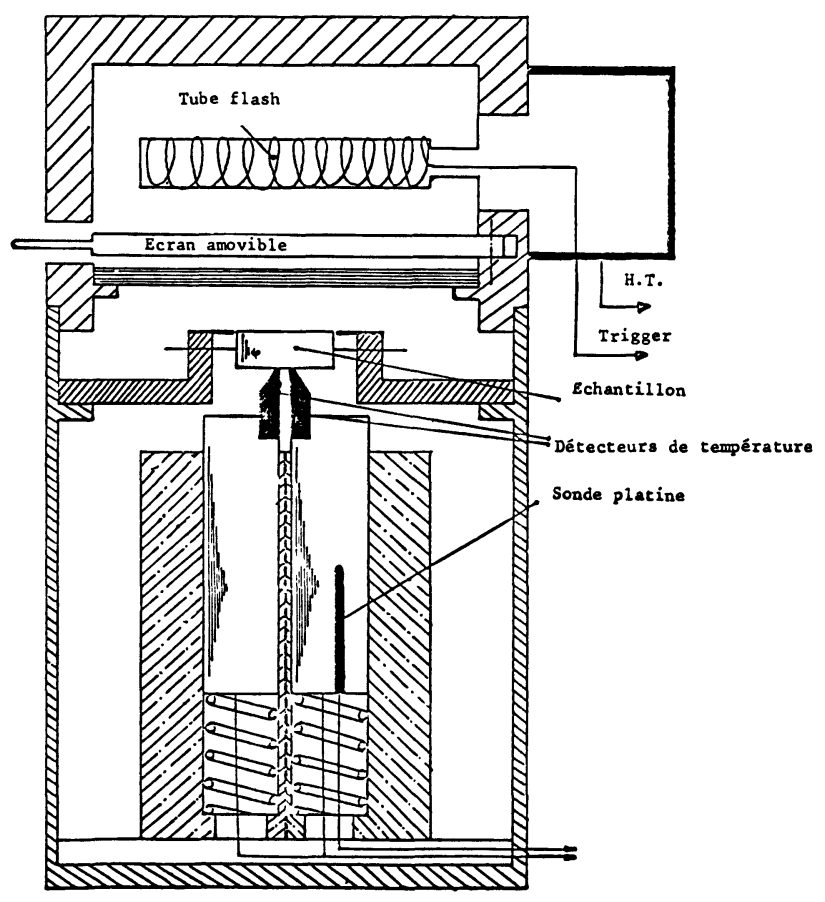

Fig. 4. - Enceinte de mesure.

[Experimental apparatus.]

Nous rappelons seulement que l'impulsion peut être assimilée à un triangle de longueur $t_{\mathrm{f}}=10 \mathrm{~ms}$ (ceci est important pour connaître les limites d'utilisation de l'appareillage). 
Nous développerons essentiellement la partie calculateur.

Le calcul de la diffusivité nécessite la mesure de $S$ et $t_{5 / 6}$, et par conséquent la connaissance de la température maximale $T_{M}$ pour effectuer la normalisation; d'où l'obligation de garder en mémoire tout le thermogramme. La partie calculateur comprendra donc :

1 amplificateur,

1 mémoire,

1 dispositif de traitement des données.

Les variations de température sont détectées par un couple à semi-conducteurs d'un pouvoir thermoélectrique d'environ $360 \mu \mathrm{V} /{ }^{\circ} \mathrm{C}$.

L'énergie fournie par le générateur permet une élévation de température maximum $T_{M}$ d'environ $1 / 3$ à $1 / 2^{\circ} \mathrm{C}$ selon les échantillons; ce qui correspond à une amplitude maximale de 100 à $200 \mu \mathrm{V}$.

La longueur de l'impulsion nous permet, sans correction [2], d'étudier des échantillons dont le temps caractéristique $\left(e^{2} / a\right)$ est supérieur à $2 \mathrm{~s}$. Ces deux contraintes permettent de définir les performances minimales de la chaîne d'amplification :

- bruit de fond ramené à l'entrée inférieur à $0,5 \mu \mathrm{V}$,

— bande passante $0-3 \mathrm{~Hz}$ à $98 \%$.

\subsection{CONDITIONNEMENT DU SIGNAL ANALOGIQUE ISSU} DU CAPTEUR DE TEMPÉRATURE. - En régime permanent, il peut exister entre le bloc isotherme et l'échantillon une différence de température fournissant une tension au maximum de $3 \mathrm{mV}$ alors que l'énergie délivrée sur le flash produit une variation de tension de $100 \mu \mathrm{V}$ à $200 \mu \mathrm{V}$ suivant les échantillons étudiés. Cette variation de tension sera numérisée à l'aide d'un convertisseur analogique numérique dont la tension d'entrée doit être comprise entre 0 et $10 \mathrm{~V}$ afin d'exploiter toute l'échelle de conversion. De ce fait il est nécessaire d'amplifier uniquement la variation de tension et ceci avec un très grand gain : $10^{4}$ à $10^{5}$.

Le dispositif de conditionnement du signal analogique est représenté sur la figure 5. L'étage d'entrée

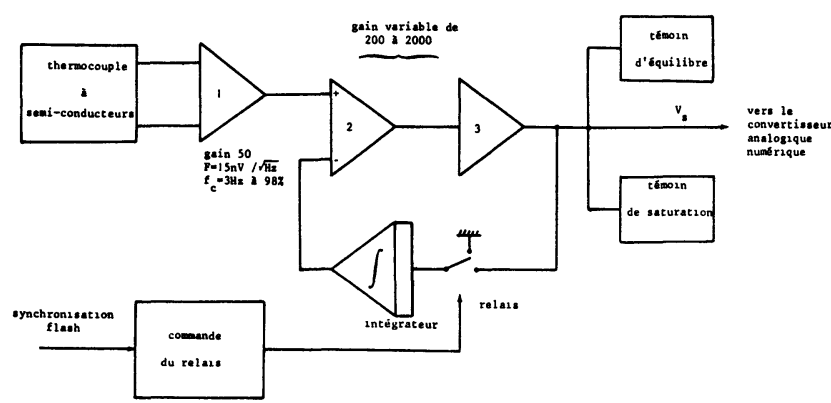

Fig. 5. - Schéma du dispositif de compensation automatique de la tension de décalage.

[Block diagramm describing the automatic compensating circuits of the offset voltage.] est un préamplificateur différentiel à faible bruit (tension de bruit ramenée à l'entrée : $15 \mathrm{nV} / \sqrt{\mathrm{Hz}}$ ) et dont la fréquence de coupure est de $3 \mathrm{~Hz}$ à $98 \%$ avec une atténuation de $20 \mathrm{db} /$ décade $(21 \mathrm{~Hz}$ à $3 \mathrm{db}$ ). Son gain est de 50. Ensuite la tension de décalage est éliminée par un dispositif à asservissement qui possède un intégrateur dans sa boucle de retour. Avant la mesure la boucle d'asservissement est fermée par un relais, ainsi la tension de sortie de l'intégrateur croît jusqu'à l'équilibre de l'asservissement, c'est-à-dire jusqu'à ce que la tension de sortie $V_{\mathrm{s}}$ du dispositif amplificateur soit nulle. Notons que nous n'avons pas à effectuer un réglage précis de la tension de décalage propre aux amplificateurs puisqu'elle est également compensée par l'asservissement.

L'équilibre de ce dispositif de compensation de la tension de décalage est indiqué par un témoin lumineux, l'opérateur peut ainsi commander l'impulsion $\mathrm{du}$ flash lorsque la compensation est effectuée. Le flash commande l'ouverture du relais qui coupe la boucle d'asservissement, l'intégrateur maintient alors sa tension de sortie qui compense la tension de décalage. Ainsi les amplificateurs 2 et 3 n'amplifient que la variation de tension provoquée par l'élévation de température de l'échantillon.

La tension $V_{\mathrm{s}}$ est numérisée, à l'aide d'un convertisseur analogique numérique de 12 bits ce qui donne une erreur de quantification négligeable $(<0,025 \%)$. Quatre fréquences d'échantillonnage sont possibles $(8 \mathrm{~Hz}, 40 \mathrm{~Hz}, 200 \mathrm{~Hz}, 1 \mathrm{kHz}$ ) permettant la mise en mémoire de thermogrammes dont la durée est comprise entre $1 \mathrm{~s}$ et $125 \mathrm{~s}$. Les valeurs numériques issues du convertisseur sont traitées par un micro-ordinateur.

3.2 Programme de Calcul de la diffusivité. Le micro-ordinateur doit faire l'acquisition des échantillons représentatifs de l'évolution de la tension délivrée par le couple thermo-électrique et transformée en valeurs binaires par le convertisseur analogiquenumérique. L'acquisition doit commencer après la réception d'un signal de synchronisation issu du dispositif de déclenchement du flash. La fréquence d'échantillonnage est fixée par l'opérateur, elle est obtenue par un oscillateur à quartz dont la fréquence est divisée pour obtenir les quatre fréquences nécessaires aux acquisitions.

Les échantillons doivent être rangés dans une mémoire, nous avons fixé leur nombre à 1000 de façon à obtenir une erreur d'intégration faible lors du calcul de la surface $S$ (relation (18)). Après la mise en mémoire des échantillons il sera possible de rechercher le mot mémoire qui contient la valeur binaire maximale. Au début de l'acquisition, des perturbations parasites induites par l'impulsion haute tension d'amorçage du flash peuvent être confondues avec des maxima. La recherche du maximum ne devra donc pas être effectuée sur les premiers échantillons. S'il n'y a pas de maximum un message d'erreur doit être 
transmis à l'opérateur. Ce message a trois causes possibles :

- la fréquence d'échantillonnage est insuffisante,

- le gain des amplificateurs est trop grand et il s'en suit une saturation,

- il y a une dérive en température de l'échantillon.

L'opérateur peut remédier à toutes ces causes d'erreurs par un réglage approprié de la fréquence d'échantillonnage, du gain et dans le cas d'une dérive, en attendant la stabilité thermique pour effectuer une nouvelle mesure. Si le maximum est atteint dès les premiers échantillons $(n<185$ dans notre programme) le calcul de $S$ sera imprécis, il est donc également nécessaire de transmettre un message d'erreur afin que l'opérateur réduise la fréquence d'échantillonnage.

Lorsque les données sont stockées dans de bonnes conditions il est alors possible de déterminer $t_{1 / 3}$ et $t_{5 / 6}$ qui sont, dans un premier temps, comptés en périodes d'échantillonnage car ces temps seront définis par des adresses de mots mémoire. La surface $S$ est alors calculée par la méthode des trapèzes. Le micro-ordinateur doit prendre connaissance de la

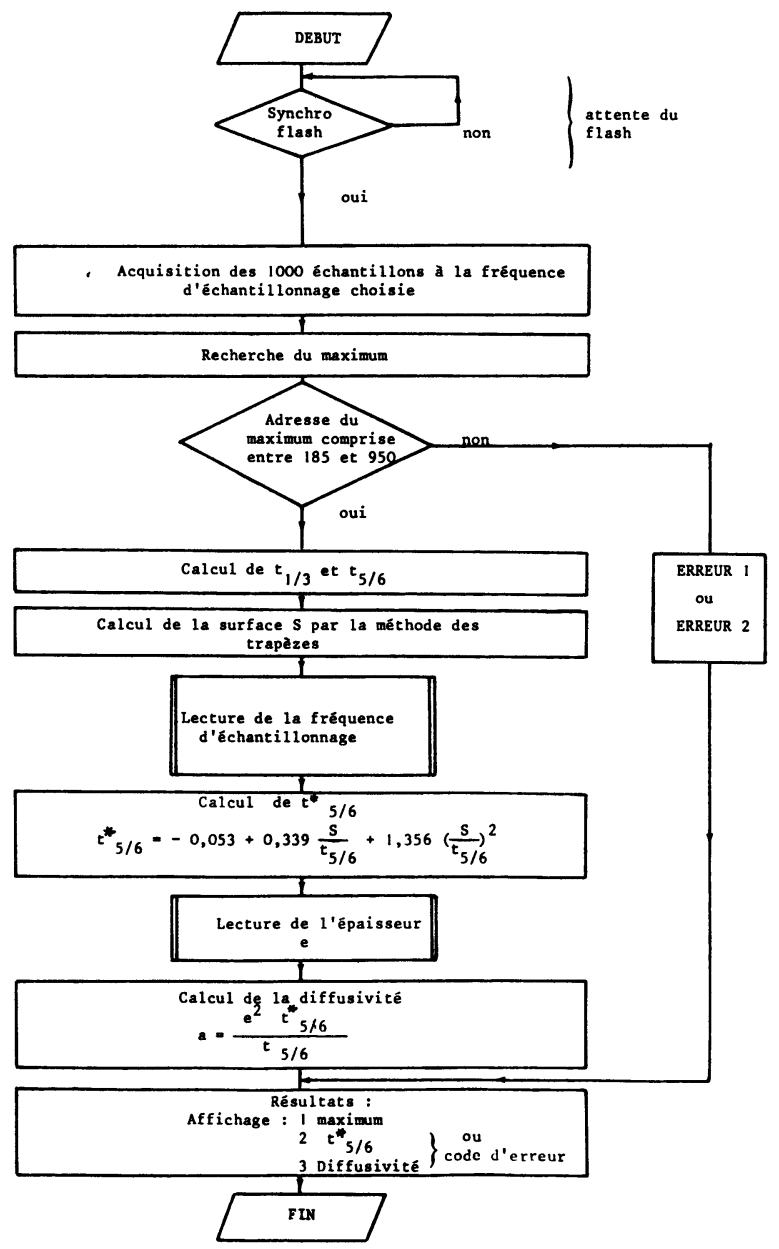

Fig. 6. - Organigramme du programme du micro-ordinateur.

[Flow chart of the microcomputer programm.] fréquence d'échantillonnage pour effectuer le calcul du temps réduit $t_{5 / 6}^{*}$ (éq. (18)), qui est communiqué à l'opérateur sur un dispositif d'affichage.

L'épaisseur de l'échantillon est introduite à l'aide de roues codeuses. Il est nécessaire d'afficher l'amplitude de l'échantillon maximum, afin de s'assurer que l'on exploite au mieux la dynamique de mesure, et évidemment d'afficher le résultat de calcul de la diffusivité.

Ces contraintes conduisent à l'organigramme de la figure 6 .

\subsection{DESCRIPTION DU MICRO-ORDINATEUR. -} L'unité centrale (CPU) du micro-ordinateur est un microprocesseur IM 6100 d'INTERSIL. Le format de ses mots de données et d'instructions est de 12 bits, ce qui permet le rangement d'un échantillon par mot mémoire. La capacité de la mémoire à accès aléatoire (RAM) est de 1,25 Kmots. Le stockage des échantillons nécessite environ $1 \mathrm{Kmots,}$, les $0,25 \mathrm{Kmots}$ supplémentaires sont utilisés par le programme. Le programme est rangé dans une mémoire morte de type REPROM, c'est-à-dire qu'il est possible d'effacer et de corriger son contenu avec un dispositif approprié. Cela permet d'envisager des aménagements du programme pour un coût financier très faible. Sa capacité est de $1 \mathrm{Kmots}$. Le programme est constitué de deux parties ; l'une est la traduction en code machine de l'organigramme de la figure 6 , l'autre est constituée par les programmes qui effectuent les opérations élémentaires : addition, multiplication, division et conversion de code. La conversion du code binaire codé décimal (BCD) au code binaire est nécessaire pour la lecture de l'épaisseur de l'échantillon dont la valeur est donnée par les roues codeuses. La conversion du code binaire au code binaire codé décimal est nécessaire pour l'affichage des différents résultats numériques.

Les opérations arithmétiques sont effectuées en virgule flottante, la mantisse est constituée de deux mots et un mot est réservé à l'exposant. Ainsi l'erreur introduite par les calculs est négligeable.

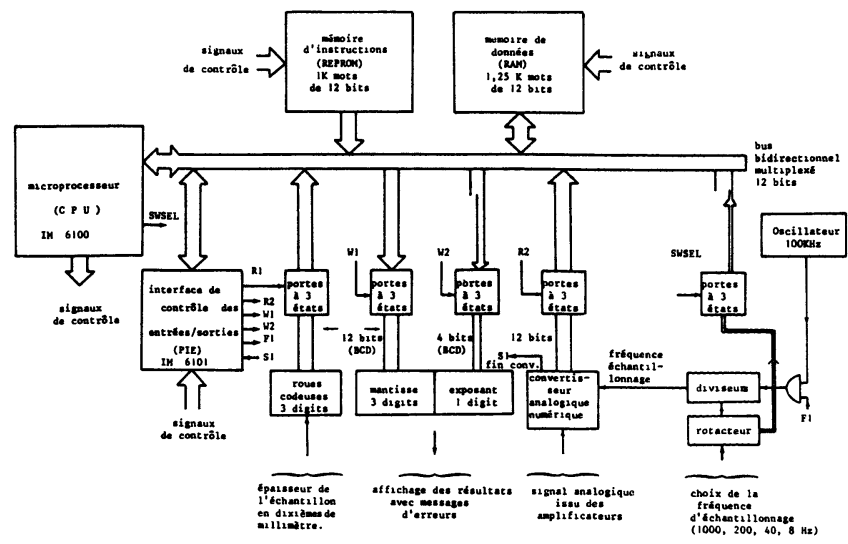

Fig. 7. - Schéma du micro-ordinateur.

[Block diagramm of the microcomputer based on a IM 6100 microprocessor.] 
Le micro-processeur possède un bus multiplexé unique de 12 bits où circulent données et instructions. Les entrées/sorties (échanges d'informations avec l'extérieur) sont assurées par un circuit spécialisé programmable (PIE). Il valide le passage des données issues du convertisseur analogique numérique, des roues codeuses, ou du sélecteur vers le bus. Il autorise également le passage des résultats du calcul, ou des messages d'erreur, vers le dispositif d'affichage.

Un schéma simplifié du micro-ordinateur est donné par la figure 7 .

4. Performances et améliorations prévues. 4.1 Performances. - Nous avons conçu la chaîne d'acquisition et le calculateur afin que leurs performances correspondent à la source d'impulsion et aux détecteurs de température. Notamment la limite minimale de l'épaisseur de l'échantillon pour les bons conducteurs est imposée par la durée de l'impulsion, soit $e^{2} / a=2 \mathrm{~s}$ (à titre indicatif cela représente une épaisseur de $15 \mathrm{~mm}$ de cuivre à température ambiante). Pour les isolants le matériau doit être suffisamment homogène pour en faire des échantillons dont le rayon est compris entre 5 et $25 \mathrm{~mm}$ et l'épaisseur entre 1 et $20 \mathrm{~mm}$.

La chaîne d'acquisition et le calculateúr peuvent être utilisés avec les dispositifs existants, par exemple impulsion laser et détection du thermogramme par rayonnement; en choisissant des éprouvettes dont les dimensions respectent les fréquences d'échantillonnage, en assurant la liaison de synchronisation entre l'impulsion et la chaîne d'acquisition et à condition que le signal provenant de la mesure des variations de température atteigne au moins $100 \mu \mathrm{V}$. Des modifications au niveau de la bande passante et de la fréquence d'échantillonnage permettraient une adaptation à tous les dispositifs existants.

Des séries de 30 essais ont montré une reproductibilité de l'ordre de $1 \%$ lorsque les conditions de stabilité thermique étaient respectées. Ce résultat constitue une amélioration vis-à-vis du traitement du signal analogique par un opérateur intervenant directement sur le thermogramme.

En affichant le maximum et $t_{5 / 6}^{*}$ on peut, en effectuant au préalable une mesure sur un échantillon connu, déterminer la chaleur volumique donc obtenir indirectement la conductivité thermique du matériau.

4.2 Améliorations PRÉVUes. - La reproductibilité des mesures sera améliorée en séparant la recherche de la température maximale de l'acquisition des points nécessaires aux calculs, et en imposant à $t_{5 / 6}$ de se situer entre le $500^{\mathrm{e}}$ et le $1000^{\mathrm{e}}$ point.

La prise en compte de la dérive avant l'impulsion permettra de réduire l'intervalle de temps entre deux expériences, il ne sera plus nécessaire d'attendre la stabilité thermique.

Une nouvelle méthode d'exploitation du modèle utilisera un grand nombre de points de référence $t_{\alpha}$ répartis sur le thermogramme.

Il sera tenu compte de la durée de l'impulsion, la limite pour l'épaisseur des échantillons sera donnée alors par :

$$
\frac{e^{2}}{a}=0,1 \mathrm{~s}
$$

5. Conclusion. - L'utilisation d'un microprocesseur pour la mesure de la diffusivité thermique facilite non seulement le travail de l'expérimentateur mais accroît également la reproductibilité des résultats.

On peut dès maintenant envisager l'élaboration de modèles thermocinétiques plus complexes se rapprochant mieux de la réalité ainsi que de nouvelles méthodes pour exploiter les thermogrammes en essayant d'utiliser au mieux les possibilités de la micro-informatique.

Remerciements. - Nous remercions les élèves du Département de Génie Electrique de l'I.N.S.A. qui ont contribué à l'élaboration de la partie électronique de ce dispositif de mesure automatique de la diffusivité thermique.

\section{Bibliographie}

[1] Poncin, H., Degiovanni, A., Laurent, M. et Lebodo, H. Thermocinétique impulsionnelle et mesure de la diffusivité thermique. Monographie du Bureau National de Métrologie (1978).

[2] Degrovanni, A., Contribution à l'étude de la diffusivité thermique. Thèse Doctorat d'Etat ès Sciences (1975).

[3] Degrovanni, A., Diffusivité et méthode flash. Conférence de la S.F.T. (1976). Revue Générale de Thermique (1977).
[4] Degiovanni, A., Gery, A., Laurent, M. et Sinicki, G., Attaque impulsionnelle appliquée à la mesure des résistances de contact et de la diffusivité thermique. Entropie $\mathrm{n}^{\circ} 64$ (1975).

[5] Degiovanni, A., Gery, A., Laurent, M., Macqueron, J. L. et SINICKI, G., Mesure de la diffusivité thermique aux moyennes températures. C.R. Acad. Sc., Paris, t. 277 (1973). 